\title{
Self-consistent analytic solution for the current and the access resistance in open ion channels
}

\author{
D. G. Luchinsky, ${ }^{1,2}$ R. Tindjong, ${ }^{1}$ I. Kaufman, ${ }^{3}$ P. V. E. McClintock, ${ }^{1}$ and R. S. Eisenberg ${ }^{4}$ \\ ${ }^{1}$ Department of Physics, Lancaster University, Lancaster LA1 4YB, United Kingdom \\ ${ }^{2}$ Mission Critical Technologies Inc., 2041 Rosecrans Avenue, Suite 225, El Segundo, California 90245, USA \\ ${ }^{3}$ VNII for Metrological Service, Gosstandart, Moscow 119361, Russia \\ ${ }^{4}$ Department of Molecular Biophysics and Physiology, Rush Medical College, 1750 West Harrison, Chicago, Illinois 60612, USA
}

(Received 10 December 2008; revised manuscript received 7 July 2009; published 21 August 2009)

\begin{abstract}
A self-consistent analytic approach is introduced for the estimation of the access resistance and the current through an open ion channel for an arbitrary number of species. For an ion current flowing radially inward from infinity to the channel mouth, the Poisson-Boltzmann-Nernst-Planck equations are solved analytically in the bulk with spherical symmetry in three dimensions, by linearization. Within the channel, the Poisson-NernstPlanck equation is solved analytically in a one-dimensional approximation. An iterative procedure is used to match the two solutions together at the channel mouth in a self-consistent way. It is shown that the currentvoltage characteristics obtained are in good quantitative agreement with experimental measurements.
\end{abstract}

DOI: 10.1103/PhysRevE.80.021925

PACS number(s): 87.16.Vy, 87.90.+y, 66.10.-x

\section{INTRODUCTION}

Ion channels are natural nanotubes in cellular membranes $[1,2]$, capable of conducting up to $10^{8}$ ions per second, selectively, through the membrane. They control a vast range of biological functions. Understanding their properties from physical first principles is a long-standing fundamental scientific problem of great interdisciplinary importance, with numerous potential applications, including, e.g., a fast growing area of research in nanofluids [3]. Theoretical treatments of ion transport through channel proteins may be classified broadly as rate theoretical approaches [4], electrodiffusion models [5], stochastic models [6], or molecular-dynamical models [7]. Of these, the first two have the advantage of providing analytic insight into the properties of ion channels over a wide range of parameters. For example, analytic approximations for the Poisson equation in the pore have been derived in [8-10]. Quasianalytic estimations of the channel current-voltage characteristics using the Poisson-NernstPlanck (PNP) theory became indispensable numerical tools in ion channel analysis because of their simplicity, physical clarity, and good quantitative agreement with experimental results [11-15]. At the same time, in most cases, the access resistance (AR) of the channel's two apertures contributes between $10 \%$ and $30 \%$ of the overall channel resistance [16] and there are channels for which the AR is believed to limit the channel's conductivity [17].

Several studies have focused on the analytic and numerical determination of the AR for ion channels. Hall [18] derived the AR by modeling the electrolyte as a homogeneous Ohmic conducting medium. Using the same considerations and by approximating the channel as a cylindrical tube, Hille [19] calculated the channel resistance by taking into account the access resistance on both sides of the channel as well as the pore resistance. This result was later extended by Sansom and Kerr [20] to consider a channel of nonuniform radius. Further insight was provided by Peskoff and Bers [21] who solved the Poisson-Boltzmann-Nernst-Planck (PBNP) equations (numerically and analytically in a number of approximations) in the bulk from infinity to a hemisphere around the channel mouth in spherical symmetry. Their calculations did not, however, combine conductivity in the bulk and in the channel. Levadny et al. [22] introduced an approximation for the AR in the hemisphere around the channel mouth assuming straight flux lines inside the hemisphere. Numerical calculations of the AR at the channel mouth are also available [23] as well as numerical solutions of the full threedimensional (3D) set of PNP equations in the channel [24] and Poisson-Boltzmann (PB) equations in the bulk and in the channel [25]. Very interesting asymptotic results for the access resistance problem of uncharged particles, where the mean time of absorption of a Brownian particle to a narrow window is calculated, were obtained in [26]. The AR problem was considered in $[13,27,28]$ where a modification of the Donnan potential formalism and the PNP model were used to obtain an approximation for the electrostatic potential at the interface between the channel and the surrounding ionic solution. This approximation is valid for channels longer than $20 \AA$ and requires a stepwise change in the excess chemical potential at the channel entrance as well as charge neutrality inside the channel.

Despite all this recent progress, an analytic solution able to connect in a self-consistent manner the ion currents and the electrostatic potentials in the access resistance region in the bulk with those in the pore is still lacking. If available, such a solution would allow for simultaneous semianalytic estimations of the AR and the current in many real ion channels, providing further physical insight into their properties. It would also allow one to set up boundary conditions (BCs) for the electric field and concentrations at the channel mouths in a self-consistent way. It is therefore very desirable to extend the theory by seeking self-consistent simultaneous solutions of the AR problem and the PBNP in the bulk, and the PNP solution in the pore.

In this paper we present such an extension, combining both solutions by means of a simple quasianalytic iterative procedure. The solution is continuous in concentration, electrostatic potential, and electric field, and it satisfies Dirichlet boundary conditions for the concentration and the potential in the bulk at plus and minus infinity. It is valid for any number of monovalent ion species and allows for simulta- 


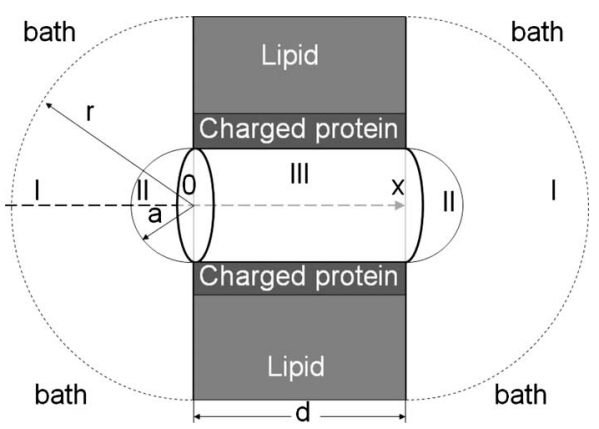

FIG. 1. Geometry of the model system under consideration. It is comprised of a cylindrical pore and two hemispheres at the left- and the right-hand mouths of the channel. The three different regions of the solution are marked as I, bulk; II, boundary hemispheres; and III, pore.

neous self-consistent estimations of the access resistance, the potential, and the current for each species.

The system considered is made of three compartments (see Fig. 1). The main compartment is a cylindrical channel in the protein, which allows ions to cross the membrane. The membrane is bathed by two solutions of different concentrations on its left- and right-hand sides. The electrodiffusion in this system is described by the Poisson equation (in SI units)

$$
\nabla \cdot\left[\varepsilon(\mathbf{r}) \nabla \widetilde{\phi}_{m}(\mathbf{r})\right]=-\sum_{i=1}^{N} z_{j} e \tilde{n}_{j}(\mathbf{r}),
$$

combined with the continuity equations for mobile ions

$$
\begin{gathered}
\frac{\partial \widetilde{n}_{j}(\mathbf{r}, t)}{\partial t}+\nabla \cdot \widetilde{J}_{j}=0 \quad(j=1, \ldots, N), \\
\widetilde{J}_{j}=-D_{j}(\mathbf{r})\left[\nabla \tilde{n}_{j}+\frac{\widetilde{n}_{j}(\mathbf{r})}{k_{B} T} \nabla \widetilde{\psi}_{j}\right] .
\end{gathered}
$$

Here, $\widetilde{\phi}_{m}(\mathbf{r})$ is the average at a given location $\mathbf{r}$ in 3D space electrostatic potential due to all mobile ions and applied electric field, $\widetilde{\psi}_{j}$ is the ion free energy, and $\widetilde{n}_{j}(\mathbf{r})$ is the number densities of mobile ions. $\widetilde{n}_{j}(\mathbf{r})=10^{3} N_{A} \widetilde{c}_{j}(\mathbf{r})$, where $\widetilde{c}_{j}(\mathbf{r})$ is the concentration (in mol/l) of mobile ion species $j$ characterized by the valence $z_{j}$, the flux $\widetilde{J}_{j}$, and the diffusion constant $D_{j} . N$ is the number of species. The Nernst-Planck equation is the sum of fluxes due to local gradients of concentration and potential. $N_{A}$ is Avogadro's number. Other parameters $\varepsilon, e, k_{B}, T$ are, respectively, the dielectric constant, the elementary charge, the Boltzmann constant, and the temperature.

Ion free energy is conveniently divided into two contributions [29]:

$$
\widetilde{\psi}_{j}(\mathbf{r})=z_{j} e \widetilde{\phi}_{m}(\mathbf{r})+\widetilde{\psi}_{j}^{P M F}(\mathbf{r}),
$$

where $\widetilde{\phi}_{m}(\mathbf{r})$ satisfy the Poisson equation (1) and $\widetilde{\psi}_{j}^{P M F}(\mathbf{r})$ is the part of the free energy associated with the potential of the mean force in the channel [30] that depends on the ion size and can be responsible of for, e.g., strong channel selectivity between alike ions [31]. In one of the obvious generaliza- tions, $\widetilde{\psi}_{j}^{P M F}(\mathbf{r})$ includes the electrostatic charge of the protein walls [24,29,32], i.e., $\widetilde{\psi}_{j}^{P M F}(\mathbf{r})=z_{j} e \widetilde{\phi}_{p}(\mathbf{r})$, where $\widetilde{\phi}_{p}(\mathbf{r})$ is the electrostatic potential due to fixed charge on the protein walls. A less obvious contribution due to the finite size of the ions and the molecules of water will be considered in details elsewhere. In the present consideration we will approximate the corresponding contribution to the free energy by a single parameter-filling factor-which is discussed in the following section.

We note that Eqs. (1) and (2) allow one to consider the modulation of the ionic flux through the channel due to, e.g., the wall vibration (cf. [33]). In the standard approximation, however, the steady-state $\left[\partial \widetilde{n}_{j}(\mathbf{r}, t) / \partial t=0\right]$ solution of the PNP equations is of interest and we arrive to the following set of equations:

$$
\begin{gathered}
\nabla \cdot[\varepsilon(\mathbf{r}) \nabla \widetilde{\phi}(\mathbf{r})]=-\sum_{i=1}^{N} z_{j} e \widetilde{n}_{j}(\mathbf{r})-e \widetilde{p}_{e x}(\mathbf{r}), \\
\nabla \cdot\left\{D_{j}(\mathbf{r})\left[\nabla \widetilde{n}_{j}+\frac{z_{j} e \widetilde{n}_{j}(\mathbf{r})}{k_{B} T} \nabla \widetilde{\phi}\right]\right\}=0,
\end{gathered}
$$

where $\widetilde{\phi}(\mathbf{r})=\widetilde{\phi}_{m}(\mathbf{r})+\widetilde{\phi}_{p}(\mathbf{r})$ and $\widetilde{p}_{e x}(\mathbf{r})$ is the number density of the partial charges fixed on the protein and the lipid atoms.

The system of equations (4) is solved in the bulk in Boltzmann approximation in three dimensions assuming spherical symmetry (the PBPN solution in region I, Fig. 1) and in the pore in one-dimensional (1D) approximation [8] (the PNP solution in region III, Fig. 1). For the PBNP solution the BCs for potential and concentration are set at \pm infinity, while the $\mathrm{BC}$ for the current and the gradient of the potential are set at the surfaces of two hemispheres of radius $a$. For the PNP solution the BCs for potential and concentration are set directly at the channel entrances. In the boundary hemispheres (region II, Fig. 1) a linear approximation (cf. [22]) is introduced in the hemispheres to ensure the continuity of the solutions. To obtain a simultaneous self-consistent solution in the bulk and in the pore, a matching procedure is developed. Using this procedure the $I-V$ characteristics are calculated for Gramicidin A (GA) channel and the results of calculations are compared with the experimental results obtained by two different groups. The access resistance found using this procedure is compared with the AR calculated from the Hall formula.

The paper is organized as follows. First, in Sec. II, we solve the 1D approximation of the PNP equations in the pore. We also consider the significance of the filling factor. Next, in Sec. III, we obtain the solution of the PBNP equations in the bulk for spherical symmetry. We demonstrate in Sec. IV that the boundary condition problem for solutions of the equations inside and outside the channel can be resolved uniquely. The joint solution is then obtained in Sec. V and compared with experimental results. In Sec. VI we analyze channel properties by investigating the influences of membrane fixed charge and bulk concentration on the permeation process. Finally, in Sec. VII, we summarize and present conclusions. 


\section{SOLUTION OF THE PNP EQUATIONS IN THE CHANNEL}

\section{A. 1D solution of the Poisson equation}

Useful insight (see, e.g., [12-15] and references therein) into the functions of many channels can be obtained from a one-dimensional approximation of Eqs. (1) and (2) within the channel, modeling it as a tube with cylindrical symmetry. Using results of $[8,9]$ the Poisson equation for a long narrow channel can be written as

$$
\begin{gathered}
-\varepsilon \frac{d^{2} \widetilde{\phi}}{d \widetilde{x}^{2}}=z_{p} e \widetilde{p}_{e x}+e \sum_{j=1}^{N} z_{j} \widetilde{n}_{j}+\widetilde{\varepsilon}\left[\tilde{\Delta}\left(1-\frac{\tilde{x}}{d}\right)-\widetilde{\phi}\right], \\
\widetilde{\phi}\left(\widetilde{x}_{0}\right)=\widetilde{\phi}_{0}, \quad \widetilde{\phi}\left(\widetilde{x}_{1}\right)=\widetilde{\phi}_{1}, \quad V_{a p p}=\widetilde{\phi}_{1}-\widetilde{\phi}_{0}=\widetilde{\Delta} .
\end{gathered}
$$

This approximation is valid for any number $N$ of ionic species in the solution. For simplicity, the dielectric constant is taken to be independent of time and space. The first term on the right-hand side represents the linear density of permanent charge on the atoms of the protein, i.e., the charge that is independent of the electric field. The total wall charge $z_{p} e$ and the parameters of the associated distribution of the permanent charge $\widetilde{p}_{e x}(x)$ are fitting parameters to be determined by comparison with experiment. The second term is the channel contents, consisting of free (mobile) charge, carried by ions in the pore as they move through the channel. The last term is the induced (i.e., polarization) charge on the protein walls of the pore [34]. The dielectric properties of the channel protein and its water-filled pore (radius $a$ and length $d)$ are described by the permittivity of free space, the (dimensionless) dielectric constants of the protein $\varepsilon_{p}$ and water $\varepsilon_{H_{2} O}$, and the effective dielectric constant of the channel $[8,9]$

$$
\widetilde{\varepsilon} \equiv \frac{\varepsilon_{p}}{\varepsilon_{\mathrm{H}_{2} \mathrm{O}}} \frac{2 \varepsilon_{0}}{a^{2} \ln (d / a)} .
$$

The constant $\widetilde{\Delta}$ is the potential difference across the channel, and the parameter $\varepsilon=\varepsilon_{0} \varepsilon_{H_{2}} \mathrm{O}$. To solve the Poisson equation we write it in dimensionless form using the following characteristic length and potential:

$$
\begin{gathered}
\widetilde{\phi}=\phi U_{T}, \quad \tilde{x}=x d, \quad U_{T}=\frac{k_{B} T}{e}, \\
\tilde{p}_{e x}=\widetilde{n}_{L}^{\infty} p_{e x}, \quad \widetilde{n}_{j}=\widetilde{n}_{L}^{\infty} n_{j} .
\end{gathered}
$$

The dimensionless number densities for the fixed and the mobile charges are introduced by normalization of $\widetilde{p}_{e x}$ and $\widetilde{n}_{j}$ as follows:

$$
\begin{gathered}
N_{f}=\widetilde{n}_{L}^{\infty} S \int_{0}^{d} \frac{d \tilde{x} \tilde{p}_{e x}(\tilde{x})}{\tilde{n}_{L}^{\infty}}=\widetilde{n}_{L}^{\infty} v_{0} \int_{0}^{1} d x p_{e x}(x), \\
N_{m}=\widetilde{n}_{L}^{\infty} S_{e f f} \int_{0}^{d} \frac{d \widetilde{x} \tilde{n}_{j}(\widetilde{x})}{\widetilde{n}_{L}^{\infty}}=\widetilde{n}_{L}^{\infty} v_{0} \int_{0}^{1} d x f_{s c} n_{j}(x),
\end{gathered}
$$

where $N_{f}$ and $N_{m}$ are the number of elementary fixed and mobile charges, $v_{0}=S d$ is the pore volume, $S$ and $S_{\text {eff }}$ are the actual and the effective cross-sectional areas for the fixed and the mobile ions, and $f_{s c}$ is the filling factor defined as $S_{e f f}=f_{s c} S$. In scaling equations (7) the bulk concentration on the far left is used as the reference concentration. The physical meaning of $S_{\text {eff }}$ is that because of their finite size and interaction with the wall, the mobile ions can fill only an effective channel cross section as will be explained in more details in Sec. II D.

The dimensionless Poisson equation has the form

$$
\begin{gathered}
\phi^{\prime \prime}-\eta^{2} \phi=-\eta^{2} \Delta(1-x)-z_{p} \alpha^{2} p-\alpha^{2} \sum_{j=1}^{N} z_{j} n_{j}, \\
\phi\left(x_{0}\right)=\phi_{0}, \quad \phi\left(x_{1}\right)=\phi_{1}, \quad \Delta=\phi_{1}-\phi_{0} .
\end{gathered}
$$

Here we have introduced two dimensionless parameters $\eta$ and $\alpha$ given by

$$
\eta^{2}=d^{2} \widetilde{\varepsilon} / \varepsilon, \quad \alpha^{2}=\frac{d^{2} e \widetilde{n}_{L}^{\infty}}{\varepsilon U_{T}} .
$$

Note that $(\alpha / d)^{-1}$ is an effective Debye length in the channel.

By a linear transformation of the potential $\phi$, Eqs. (8) can be transformed into a zero boundary value problem. The resultant solution is given by

$$
\begin{gathered}
\phi(x)=\frac{\left(\phi_{1}-\phi_{0}\right)}{\left(x_{1}-x_{0}\right)}\left(x-x_{0}\right)+\phi_{0}+\int_{x_{0}}^{x_{1}} G(x, s) f(s) d s, \\
f(s)=\eta^{2}\left[\frac{\left(\phi_{1}-\phi_{0}\right)}{\left(x_{1}-x_{0}\right)}\left(s-x_{0}\right)+\phi_{0}-\Delta(1-s)\right]-\alpha^{2} z_{p} p(s) \\
\quad-\alpha^{2} \sum_{j=1}^{N} z_{j} n_{j}(s),
\end{gathered}
$$

$G(x, s)= \begin{cases}A \sinh \eta\left(s-x_{1}\right) \sinh \eta\left(x-x_{0}\right), & x_{0} \leq x \leq s \\ A \sinh \eta\left(s-x_{0}\right) \sinh \eta\left(x-x_{1}\right), & s \leq x \leq x_{1} .\end{cases}$

Here, $G(x, s)$ is the associated Green's function and $A$ $=1 /\left[\eta \sinh \eta\left(x_{1}-x_{0}\right)\right]$. The permanent charge is modeled using a unimodal or a multimodal Gaussian distribution, consistent with the fact that it is induced by local charges on the channel wall $[35,36]$,

$$
p(x)=\sum_{i=1}^{M} \frac{1}{\sqrt{2 \pi \sigma_{i}^{2}}} \exp \left(-\frac{\left(x-\mu_{i}\right)^{2}}{2 \sigma_{i}^{2}}\right),
$$

where $\mu_{i}$ and $\sigma_{i}$ are the mean value and the variance of the coordinate of the $i$ th wall charge. The parameters of this distribution, together with the parameters of the axial distribution of the diffusion coefficient for each ion species, are the fitting parameters of the model. Having estimated the solution of the Poisson equation, we now focus on the Nernst-Planck equation for determination of the current density and the concentrations of the two ion species.

\section{B. Solution of the Nernst-Planck equation in the channel}

The problem to be discussed in this section is the one described by Eisenberg et al. [37]. The local concentration of ions of species $j$ located on the axis between $\tilde{x}_{0}=0$ and 
$\widetilde{x}_{1}=d$ satisfies the Nernst-Planck equation in the Stratonovich form [38]

$$
\frac{d}{d \widetilde{x}} D_{j}\left[\frac{d \widetilde{n}_{j}(\widetilde{x})}{d \widetilde{x}}+\frac{z_{j} e}{k_{B} T} \widetilde{n}_{j}(\widetilde{x}) \frac{d \widetilde{\phi}}{d \widetilde{x}}\right]=0 \quad \text { for } \widetilde{x}_{0} \leq \tilde{x} \leq \widetilde{x}_{1},
$$

which is the 1D form of Eq. (2). The diffusion coefficient $D_{j}(\widetilde{x})$ is taken to be a function of the position of the ion on the channel axis. Taking into account the normalization conditions (7) and the corresponding scaling for the effective cross-sectional area, the concentrations at the channel mouth result in the following boundary conditions for Eq. (11):

$$
\tilde{n}_{j}\left(\widetilde{x}=\widetilde{x}_{0}\right)=\tilde{n}_{j L}, \quad \tilde{n}_{j}\left(\widetilde{x}=\widetilde{x}_{1}\right)=\tilde{n}_{j R} .
$$

Integrating Eq. (11) once, we obtain

$$
\widetilde{J}_{j}=-D_{j}\left[\frac{d \tilde{n}_{j}(\tilde{x})}{d \tilde{x}}+\frac{z_{j} e}{k_{B} T} \tilde{n}_{j}(\widetilde{x}) \frac{d \widetilde{\phi}}{d \tilde{x}}\right],
$$

where $\tilde{J}_{j}$ is the current density carried by ions $j$ (i.e., the current per unit area, corresponding to the flux of ions $j$ through the channel). We now rewrite this equation in terms of dimensionless variables. Using the scaling factors from Eqs. (6) and (7), the dimensionless Nernst-Planck equation is therefore given by

$$
\begin{gathered}
J_{j}^{c h}=\frac{\widetilde{J}_{j} d}{D_{j} \widetilde{n}_{L}^{\infty}}=-\left[\frac{d n_{j}(x)}{d x}+n_{j}(x) \frac{d z_{j} \phi}{d x}\right], \\
n_{j}\left(x=x_{0}\right)=n_{j L}, \quad n_{j}\left(x=x_{1}\right)=n_{j R} .
\end{gathered}
$$

Integrating Eqs. (14), the analytic flux and the concentration can [37] be calculated as

$$
\begin{gathered}
J_{j}^{c h}=\frac{n_{j L} e^{z_{j} \phi_{0}}-n_{j R} e^{z_{j} \phi_{1}}}{\int_{x_{0}}^{x_{1}} e^{z_{j} \phi(s)} d s}, \\
n_{j}(x)=e^{-z_{j} \phi(x)}\left[n_{j L} e^{z_{j} \phi_{0}}-J_{j}^{c h} \int_{x_{0}}^{x} e^{z_{j} \phi(s)} d s\right] .
\end{gathered}
$$

The solution of the Poisson equation and the current density coupled to the ionic concentrations may now be calculated simultaneously in a self-consistent manner. The fitting parameters are the diffusion coefficients $D_{j}$ together with the parameters describing the distribution of fixed charge on the channel walls. The ionic currents $\widetilde{J}_{j}$ inside the channel and the total current are given by

$$
\tilde{I}_{j}=z_{j} e S_{e f f} \tilde{J}_{j}=\frac{z_{j} e D_{j} \tilde{n}_{L}^{\infty} S_{e f f}}{d} J_{j}^{c h}, \quad \tilde{I}=\sum_{j=1}^{N} \tilde{I}_{j} .
$$

\section{Self-consistent solution of PNP equations in the channel}

A self-consistent (simultaneous) solution of the PNP equations (8) and (14) can be obtained using the well-known Gummel [39] iterative procedure from semiconductor physics, ensuring that the Poisson equation and the far-field boundary conditions are always satisfied [2]. As a first step we calculate the concentrations making a linear initial guess for the potential
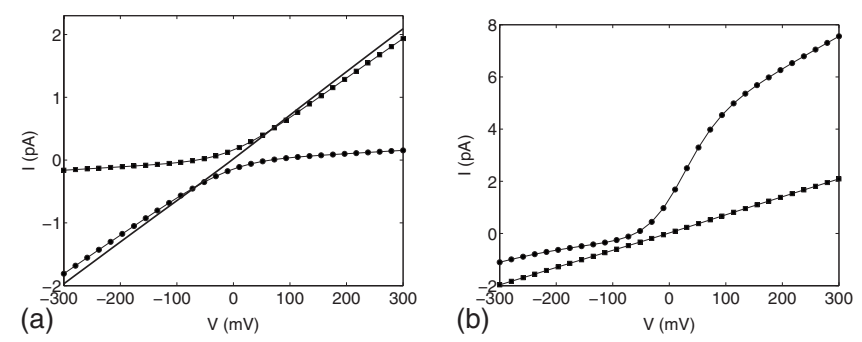

FIG. 2. (a) Calculated current-voltage $(I-V)$ characteristics for the uncharged GA channel. The thick line shows the total current, the squares represent the positive ion current, and the filled circles represent the negative ion current. The thinner lines joining calculated points are guides to the eye. Ionic concentrations in the lower (or left) and upper (or right) reservoirs are $c_{L}=500 \mathrm{mM}$ and $c_{R}$ $=40 \mathrm{mM}$, respectively. Diffusion constants are $D_{+}=D_{-}=1.27$ $\times 10^{-10} \mathrm{~m}^{2} / \mathrm{s}$. To fit the result, we used a channel of radius $=4 \AA$ and length $=30 \AA$ with a filling factor of 0.16 . (b) The corresponding current-voltage characteristics for charged GA (circles) with a filling factor of 0.28 and uncharged GA (squares).

$$
\phi(x ; \text { initial guess })=\frac{\left(\phi_{1}-\phi_{0}\right)}{\left(x_{1}-x_{0}\right)}\left(x-x_{0}\right)+\phi_{0} .
$$

Using the given concentrations and electrostatic potential in the bulk, we then set boundary conditions directly at the channel boundaries. Next, we obtain concentrations and currents using Eqs. (15)-(17). The concentrations obtained are used to update the value of the potential. This procedure can be repeated to obtain a higher-order accuracy of the solution. Note that the linear part of the potential $\phi(x)$ in Eq. (9), the Green's function (9), and the two first terms in function (9) are fixed and have to be calculated only once during the iterations. This numerical procedure was used earlier by Eisenberg [40]. The difference in the present calculation is that we solve the Poisson equation analytically and then couple it to the solution of the access resistance equations (see below).

The output of these calculations is the axial distributions of the electrostatic potential $\widetilde{\phi}(x)$ and of the ions $\widetilde{c}_{j}(x)$, the current $\widetilde{I}_{j}$ for species $j$, and the total current $\widetilde{I}$ as a function of the applied voltage and the ionic concentrations in the bulk solutions.

In Fig. 2(a) we present our calculation of the resultant current-voltage characteristic for an uncharged channel. It can be seen that, in the absence of a net charge on the wall, the $I-V$ characteristic is linear. The effect of a net charge on the current is analyzed in Fig. 2(b) where we compare the $I-V$ characteristics for uncharged and charged channels. The net negative wall charge used for this calculation is equal to $-0.2 e$. It is distributed on the membrane walls using a twomode Gaussian distribution with mean values $\mu_{j}$ equal to $1 / 6$ near the channel mouth and $1 / 2$ at the center of the channel. The variance is chosen as $\sigma=0.1$. The results shown in Fig. 2(b) are obtained with the same values of parameters for the channel geometry, diffusion coefficients, and concentrations as in [24] and are in good qualitative agreement with both the results of full 3D solutions of the PNP equations and with experimental measurements as compared to Figs. 5 and 6 of 
[24]. These results demonstrate that a simple calibration of the analytic solution allows one to get the same result as yielded by the full 3D simulations.

\section{Filling factor}

We note that the current in the $I-V$ characteristics shown in Fig. 2 is calculated as $I=e \widetilde{J} S_{c h}$, where $\widetilde{J}$ is the current density obtained using the self-consistent solution of the PNP equations and $S_{c h}$ is the channel's cross-sectional area. We note further that the continuous approximation introduced above is valid only on the channel axis. Away from the channel axis the current density has to decay rapidly for a number of reasons. First, the ions that carry the current cannot come closer then their own radii to the channel wall. Second, the effective channel radius is further reduced due to the presence of dielectric walls of low dielectric constant. Indeed, the charges induced by an ion on the dielectric walls force the ion to stay near the channel axis, so that the probability of finding the ion at a finite distance $r$ from the axis decays rapidly with increasing $r$. Moreover, the actual geometry of the channel is complicated (e.g., the channel is not cylindrical) and the channel can only be characterized by an effective radius averaged over the channel length. For these reasons, calculation of the total current through the channel using the axial current density $\widetilde{J}$ requires one to introduce an effective cross-sectional area of the channel $S_{c h}=S_{e f f}$ $=\pi a^{2} f_{s c}=\pi a_{e f f}^{2}$ (see also [41]).

The filling factor may have a broader physical significance, and it can be used to compensate for inhomogeneity related to all the space-dependent parameters of the system that are being neglected in the cylindrical symmetry approximation. We note that this factor is introduced as a simple approximation of the free-energy contribution due to the potential of the mean force in the channel. We note also that factors of this type are common in semiconductor theories (cf. the ideality factor in the diode equation [42]) used to model conduction in ion channels [33]. The adjustment of the effective cross-sectional area of the channel in an analytic 1D approximation allows one to obtain qualitatively good agreement with the full 3D PNP solution of Kurnikova et al. [24], whose results fit experiments. Therefore, taking into account earlier examples of the successful application of the 1D approximation (see, e.g., [11-15]) our analytic solution can be calibrated with a suitably chosen filling factor and then used as a convenient tool for obtaining simple analytic estimates of the fundamental conducting properties of many ion channels.

The 1D approximation obtained above can be further improved by taking into account changes in the potential and the ion concentrations in the bulk. The latter difference can be estimated using, e.g., the Donnan potential or the access resistance formalism as discussed in the Introduction. In what follows we solve the problem using the access resistance formalism by coupling the PNP solution in the channel (discussed in this section) to the analytic solution of the PBNP equations in the bulk (next section) in a self-consistent manner.

\section{SOLUTION OF THE PBNP EQUATIONS IN THE BULK}

To find an analytic solution of the PBNP equations in the bulk in spherical symmetry, we follow closely the considerations of Peskoff and Bers [21]. As already mentioned, the solution in bulk spans from $\pm \infty$ to a hemisphere at each channel mouth of radius equal to the effective radius of the channel.

\section{A. Nernst-Planck equation in spherical symmetry}

Consider first a solution of the Nernst-Planck equation (2), rewrite it in spherical symmetry as

$$
\tilde{J}_{j}=-D_{b, j}\left[\frac{d \tilde{n}_{j}(\widetilde{r})}{d \widetilde{r}}+\frac{z_{j} e}{k_{B} T} \tilde{n}_{j}(\widetilde{r}) \frac{d \tilde{\phi}}{d \widetilde{r}}\right],
$$

and set the following boundary conditions at infinity:

$$
\widetilde{n}_{j}(\widetilde{r}=\infty)=\widetilde{n}_{j}^{\infty}, \quad \widetilde{\phi}(\widetilde{r}=\infty)=\widetilde{\phi}^{\infty} .
$$

And at the surface of the semisphere closed to the channel mouth

$$
\tilde{J}_{j}\left(\tilde{r}=a_{e f f}\right)= \pm \frac{\tilde{I}_{j}}{2 e \pi a_{e f f}^{2}} .
$$

Here, $a_{e f f}$ is the effective radius of the channel and $\tilde{I}_{j}$ is the current of the $j$ th ion found in the solution of the PNP equations above inside the channel. The "-" sign is for ions in the left-hand bath and the "+" sign is for ions in the right-hand bath. We use the following scaling factors for the electric potential, the radial coordinate, and the ion concentration:

$$
\widetilde{\phi}=\phi U_{T}, \quad \tilde{r}=r d, \quad \tilde{n}_{j}=n_{j} \tilde{n}_{L}^{\infty} .
$$

The dimensionless form of Eq. (19) can then be written as

$$
J_{j}=\frac{\tilde{J}_{j} d}{D_{b, j} \tilde{n}_{L}^{\infty}}=-\left[\frac{d n_{j}(r)}{d r}+n_{j}(\widetilde{r}) \frac{d z_{j} \phi}{d r}\right] .
$$

Integration of Eq. (19) with the boundary conditions (20) and (21) can be performed in a way similar to integration of Eq. (14), taking into account that $\widetilde{J}_{j}(\widetilde{r})= \pm \widetilde{I}_{j} / 2 e \pi \widetilde{r}^{2}$. We thus obtain

$$
\begin{gathered}
n_{j}(r)=\left[\frac{\tilde{n}_{j}^{\infty}}{\tilde{n}_{L}^{\infty}} e^{z_{j} \phi^{\infty}} \mp \beta_{j} \int_{r}^{\infty} d s \frac{e^{z_{j} \phi(s)}}{s^{2}}\right] e^{-z_{j} \phi(r)}, \\
\beta_{j}=\frac{\tilde{I}_{j}}{2 e \pi \tilde{n}_{L}^{\infty} D_{b, j} d}=\left(\frac{a_{e f f}}{d}\right)^{2}\left(\frac{D_{j}}{2 D_{b, j}}\right) J_{j}^{c h} .
\end{gathered}
$$

This solution can be further simplified if we neglect the change in potential in the bulk to obtain

$$
n_{j}(r)=n_{j}^{\infty} \mp \frac{\beta_{j}}{r} .
$$

Note the difference in sign of this solutions arising from the difference in the radial directions in the left and the right sections of the system (see Fig. 1). 


\section{B. Poisson-Boltzmann equation in spherical symmetry}

Solution (24) depends on the electrostatic potential $\widetilde{\phi}(\widetilde{r})$, which can in turn be determined by solution of the PoissonBoltzmann equation. In spherical coordinates, for $N$ ion species, this can be written as

$$
\begin{gathered}
\varepsilon \frac{1}{\widetilde{r}} \frac{d^{2}}{d \widetilde{r}^{2}}(\widetilde{r} \widetilde{\phi})=-e \sum_{j=1}^{N} z_{j} \widetilde{n}_{j}, \\
\widetilde{\phi}(\widetilde{r}=\infty)=\widetilde{\phi}_{L}^{\infty}, \quad \nabla \widetilde{\phi}(\widetilde{r}=a)=\nabla \widetilde{\phi}^{P N P}\left(\widetilde{x}=\tilde{x}_{0}\right),
\end{gathered}
$$

where $\varepsilon=\varepsilon_{\mathrm{H}_{2} \mathrm{O}} \varepsilon_{0}$ is the product of the water dielectric constant and the electric permittivity of the vacuum and $\widetilde{\phi}^{P N P}$ is the PNP solution obtained inside the channel as calculated in Sec. II. At infinity, away from the channel mouth, the solution is assumed to be charge neutral $\left(\sum_{j=1}^{N} z_{j} \tilde{n}_{j}^{\infty}=0\right)$. Note, however, that neutrality is not required at the channel mouth, unlike in the Donnan potential formalism [27]. Using the dimensionless variables defined in Eq. (8), and substituting the solution of the Nernst-Planck equation (23) into Eq. (27), we obtain the following integrodifferential equation for monovalent ion solutions:

$$
\begin{aligned}
\frac{1}{2 \alpha^{2} r} \frac{d^{2}}{d r^{2}}(r \phi)= & \sinh \left[\phi(r)-\phi_{L}^{\infty}\right] \\
& +\frac{1}{2} \sum_{j} z_{j} n_{j}^{\infty} \beta_{j} e^{-z_{j} \phi(r)} \int_{r}^{\infty} \frac{e^{z_{j} \phi(s)}}{s^{2}} d s .
\end{aligned}
$$

We have assumed for simplicity that the diffusion coefficients do not depend on $r$, although this simplification can be readily lifted if necessary. The solution of the integrodifferential equation is not trivial. It can be solved numerically to determine the electrostatic potential. However, it was shown by Peskoff and Bers [21] that the solution found in a linear approximation of the $\mathrm{PB}$ equation agrees well with the exact solution under most physiological conditions. Therefore, we linearize the PB equation for small values of the electrostatic potential and obtain a reduced equation in the form [21]

$$
\frac{1}{2 \alpha^{2} r} \frac{d^{2}}{d r^{2}}(r \phi)=\phi(r)-\phi_{L}^{\infty}+\frac{\beta}{r},
$$

where $\beta=\frac{1}{2} \sum_{j} z_{j} \beta_{j}$. Integration of Eq. (30) yields the following expression for the electrostatic potential:

$$
\phi(r)=\frac{\left(\beta+S_{0}\right) e^{\sqrt{2} \alpha\left(a_{e f f} / d-r\right)}}{\left(1+\sqrt{2} \alpha a_{e f f} / d\right) r}-\frac{\beta}{r}+\phi_{L}^{\infty},
$$

where $S_{0}=\left(a_{e f f} / d\right)^{2} \nabla \phi^{P N P}\left(x_{0}\right)$ is proportional to the gradient of the electrostatic potential in the channel determined in the previous section from the self-consistent solution of the PNP equations. Note that the signs of the potential gradient and the current in Eq. (31) are determined by the fact that the directions of the radial coordinate $r$ and the axial coordinate $x$ in Eqs. (8) and (19) in the left bulk are opposite.

Similarly, the bulk solution for the electrostatic potential on the right-hand side can be written by making sure that the sign of the current density Eq. (21) is properly chosen,

$$
\phi(r)=-\frac{\left(\beta_{f}+S_{1}\right) e^{\sqrt{2 n_{f}} \alpha\left(a_{e f f} / d-r\right)}}{\left(1+\sqrt{2 n_{f}} \alpha a_{e f f} / d\right) r}+\frac{\beta_{f}}{r}+\phi_{R}^{\infty} .
$$

where $\quad S_{1}=\left(a_{e f f} / d\right)^{2} \nabla \phi^{P N P}\left(x_{1}\right), \quad n_{f}=\tilde{n}_{R}^{\infty} / \tilde{n}_{L}^{\infty}, \quad$ and $\quad \beta_{f}$ $=\left(1 / 2 n_{f}\right) \sum_{j} z_{j} \beta_{j}$. The factor $n_{f}$ appears because we scale concentrations on both sides of the channel by the same value $\tilde{n}_{L}^{\infty}$.

Equations (24), (31), and (32) provide the required solution of the PBNP equations in the bulk. To determine spatial distributions of the concentrations $n_{j}(r)$ and the electrostatic potential $\phi(r)$ (and their values at the channel mouth) in a self-consistent manner, these equations can be solved simultaneously using an iterative procedure analogous to that introduced in the previous section for the PNP solution.

Note that these solutions depend on the concentrations and the electrostatic potential at infinity, the dimensionless current densities $\beta_{j}$, and the gradient of electrostatic potential $\nabla \phi^{P N P}$ at both mouths of the channel. The latter values are provided by the PNP solution in the channel. In turn the PNP solution is determined by the concentrations and the electrostatic potential at the channel mouth provided by the PBNP solution. This feature allows one to couple the PBNP solution in the bulk to the PNP solution in the channel in a self-consistent way, as described in the next sections.

\section{LINEAR APPROXIMATION}

To appreciate that the boundary condition problem can be resolved uniquely, let us consider a linear approximation of the joint solutions (9), (16), (26), (31), and (32). We note that, when both ion concentrations and fixed charge in the channel are relatively small, the last term in Eqs. (9) can be neglected. In this approximation $S_{1}=S_{0}=r_{e f f}^{2} \nabla \phi^{P N P}, \nabla \phi^{P N P}$ $=\left(\phi_{1}-\phi_{0}\right) /\left(x_{1}-x_{0}\right), r_{e f f}=a_{e f f} / d$, and $x_{1}-x_{0}=1$ in dimensionless coordinates. To simplify this discussion further, we neglect the width of the boundary layers. The resulting set of equations determines the boundary conditions as

$$
\begin{gathered}
\phi_{1}=\phi_{R}^{\infty}-\frac{r_{e f f} \frac{\phi_{1}-\phi_{0}}{x_{1}-x_{0}}-\left(\sum_{j=1}^{N} z_{j} \beta_{j}\right) \alpha / \sqrt{2 n_{f}}}{1+\sqrt{2 n_{f}} \alpha r_{e f f}}, \\
\phi_{0}=\phi_{L}^{\infty}+\frac{r_{e f f} \frac{\phi_{1}-\phi_{0}}{x_{1}-x_{0}}-\left(\sum_{j=1}^{N} z_{j} \beta_{j}\right) \alpha / \sqrt{2}}{1+\sqrt{2} \alpha r_{e f f}}, \\
\beta_{j}=r_{e f f}^{2} \frac{D_{j}}{2 D_{b, j}} J_{j}^{c h}=\frac{z_{j}\left(\phi_{1}-\phi_{0}\right) r_{e f f}^{2}}{x_{1}-x_{0}} \frac{D_{j}}{2 D_{b, j}} \frac{n_{j L}-n_{j R} e^{z_{j}}\left(\phi_{1}-\phi_{0}\right)}{e^{z_{j}\left(\phi_{1}-\phi_{0}\right)}-1},
\end{gathered}
$$

where $n_{j L}$ and $n_{j R}$ are given by Eq. (26) at the surfaces of the boundary hemispheres $n_{j L, R}=n_{j L, R}^{\infty} \mp\left(\beta_{j} / r\right)$.

This is the system of $N+2$ equations with $N+2$ unknowns ( $N$ unknown $\beta_{j}$ and two unknown potentials at the channel entrances $\phi_{1}$ and $\phi_{0}$ ) and has a unique solution that allows for the simultaneous solution of the access resistance prob- 
lem and coupled Poisson and Nernst-Planck equations in the channel and in the bulk. Now, we describe the algorithm for simultaneous solution of these equations in the more general nonlinear case.

\section{JOINT SOLUTION}

In order to complete the calculation of ion density and electrostatic potential everywhere in space, we couple the PNP solution in the channel and the PBNP solution in the bulk. The bulk concentrations and the electric potential for the coupled solution are set at infinity. Near the channel mouth, on the surface of the hemisphere, the boundary conditions are given by the continuity conditions for the electric field and the current. The joint set of equations has the form

$$
\begin{gathered}
\phi(x)=\frac{\left(\phi_{1}-\phi_{0}\right)}{\left(x_{1}-x_{0}\right)}\left(x-x_{0}\right)+\phi_{0}+\int_{x_{0}}^{x_{1}} G(x, s) f(s) d s, \\
J_{j}^{c h}=\frac{n_{j L} e^{z_{j} \phi_{0}}-n_{j R} e^{z_{j} \phi_{1}}}{\int_{x_{0}}^{x_{1}} e^{z_{j} \phi(s)} d s}, \\
n_{j}(x)=e^{-z_{j} \phi(x)}\left[n_{j L} e^{z_{j} \phi_{0}}-J_{j}^{c h} \int_{x_{0}}^{x} e^{z_{j} \phi(s)} d s\right], \\
n_{j}(r)=n_{j}^{\infty} \mp \frac{\beta_{j}}{r}, \\
\phi(r)=\frac{\left(\beta+S_{0}\right) e^{\sqrt{2} \alpha\left(a_{e f f} / d-r\right)}}{\left(1+\sqrt{2} \alpha a_{e f f} / d\right) r}-\frac{\beta}{r}+\phi_{L}^{\infty}, \\
\phi(r)=-\frac{\left(\beta_{f}+S_{1}\right) e^{\sqrt{2 n_{f}} \alpha\left(a_{e f f} / d-r\right)}}{\left(1+\sqrt{2 n_{f}} \alpha a_{e f f} / d\right) r}+\frac{\beta_{f}}{r}+\phi_{R}^{\infty} .
\end{gathered}
$$

This leads us to the following algorithm for obtaining the self-consistent solution:

(1) Initialization. Set the bulk values of the concentrations and the electrostatic potential on the left- and the right-hand sides of the channel $c_{L}^{\infty}, \phi_{L}^{\infty}$ and $c_{R}^{\infty}, \phi_{R}^{\infty}$, respectively. Set the potential to its initial value given by the linear function in Eq. (18).

(2) PNP solution in the channel. (a) Calculate concentrations in the channel using Eqs. (15) and (16), (b) update the value of the potential using Eq. (9), (c) repeat calculations from step (2a) until convergence is reached, (d) determine the currents inside the channel for each ion species, and calculate the electric field at the channel mouth.

(3) PBNP solution in the bulk. (a) Update the value of the potential using Eq. (31), (b) calculate concentrations in the bulk using Eq. (24), and (c) use linear approximation in the boundary layer to determine the concentration and the potential at the channel mouth.

(4) Iteration. Repeat steps (2) and (3) in turn until convergence is reached.

The solution obtained in the previous section for the current and the potential gradient in the pore is thus used to set

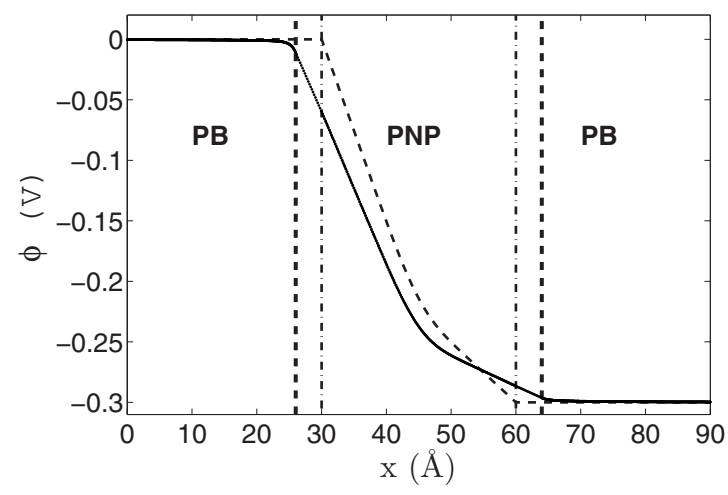

FIG. 3. Electrostatic potential in the model, calculated for the following boundary conditions: $\phi(-\infty)=\phi_{L}^{\infty}=0, \quad \phi(\infty)=\phi_{R}^{\infty}=$ $-300 \mathrm{mV}, c(-\infty)=c_{L}^{\infty}=500 \mathrm{mM}, c(\infty)=c_{R}^{\infty}=40 \mathrm{mM}$, and fixed charge $-0.227 e$ in the middle of the channel normally distributed with standard deviation $\sigma=0.1$. Diffusion constants are $D_{\mathrm{Na}^{+}}$ $=D_{\mathrm{Cl}^{-}}=2.09 \times 10^{-10} \mathrm{~m}^{2} / \mathrm{s}$. The initial solution for the PNP equations in the channel is shown by the thin dashed line. The result of the self-consistent calculations of the potential in the bathing solutions and in the pore is shown by the thick solid line. The vertical thin dashed-dotted lines indicate the positions of the channel entrances. The vertical thick dashed lines show the boundaries for the calculations of the access resistance.

up boundary conditions for the PBNP equations in the bath. In turn, solutions (24) and (31) can be used to set up the boundary conditions for the PNP equations in the pore. The convergence of the successive approximations is guaranteed by the fact that, in the zeroth approximation, the current through the channel and the electric field at the channel mouth have maximum possible values. At the next step this leads to the maximum possible reduction in the concentration and the potential at the channel mouth. In turn, this reduces the current and the electric field within the channel, which then leads to a smaller correction at the channel mouth, i.e., the scheme converges, leading to the solution shown in Fig. 3. Note that, in the two regions of thickness $a_{\text {eff }}$ (radius of the channel) on the left- and the right-hand sides of the channel, the gradient of the electric potential is assumed to be constant and equal to that in the channel. The corresponding density profile for two ionic species is presented in Fig. 4. As expected, the density profiles show a significant rise for $\mathrm{Na}^{+}$and an abrupt decrease for $\mathrm{Cl}^{-}$inside the channel. This suggests that the channel is most of the time occupied by a single $\mathrm{Na}^{+}$ion but almost zero occupancy of $\mathrm{Cl}^{-}$ions, on average; we confirm this later when we estimate the channel occupation number as a function of the membrane fixed charge and the bulk concentration.

Another possible way of connecting the solution inside the channel to that in the bulk would be by matching the solutions asymptotically [33]. Although in a sense more interesting from the theoretical point of view, its use here would neither clarify our approach nor improve the quality of the fits to experimental results. It could, however, form the basis of a future project building on the ideas presented in the present paper. 


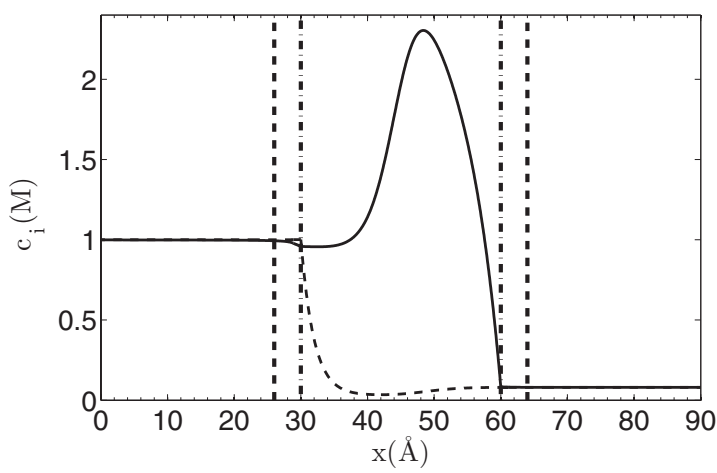

FIG. 4. Ion density profiles in the model, calculated for the following boundary conditions: $\phi(-\infty)=\phi_{L}^{\infty}=0, \quad \phi(\infty)=\phi_{R}^{\infty}=$ $-300 \mathrm{mV}, n(-\infty)=n_{L}^{\infty}=500 \mathrm{mM}, n(\infty)=n_{R}^{\infty}=40 \mathrm{mM}$, and fixed charge $-0.227 e$ in the middle of the channel normally distributed with standard deviation $\sigma=0.1$. Diffusion constants are $D_{\mathrm{Na}^{+}}$ $=D_{\mathrm{Cl}^{-}}=2.09 \times 10^{-10} \mathrm{~m}^{2} / \mathrm{s}$. The vertical thin dashed-dotted lines indicate the position of the channel entrances. The vertical thick dashed lines show the boundaries for the calculations of access resistance.

\section{Comparison with the experiments}

The model is capable of reproducing experimental measurements if appropriate fitting parameters are used. These parameters include the distribution and the magnitude of the channel wall's fixed charge, the ionic diffusion coefficient in the bulk and inside the channel, and the filling factor described in Sec. II D. The fixed negative charge on the membrane wall is taken equal to $-0.227 e$. This corresponds to the number of particles inside the channel with a concentration of $250 \mathrm{mM}$. We stress that the projection of the distribution of fixed charge on the channel axis for the 1D approximation derived from the known 3D distribution of fixed charge has yet to be determined. The fixed charge is distributed along the 1D channel axis using a multimodal Gaussian distribution. With a suitable choice of these parameters, it is possible to fit experimental measurements for different concentrations.

Figure 5 compares the $I-V$ (current-voltage) characteristics with experimental measurements by Oiki et al. [43] in a $1 \mathrm{M} \mathrm{CsCl}$ solution. The calculated current is given by the black thick line, while the experimental measurements are given by the circles. The calculated current is in good agreement with the experimental measurement. The robustness of this method is further tested by comparison with another set of experimental results presented by [44], as shown in Fig. 6. Again, good agreement is obtained between experiment and theory. Here, the $(I-V)$ characteristics in the model are calculated for a channel with $-0.227 e$ fixed charge distributed using a three-mode Gaussian distribution. The fixed charge weights, the positions, and the standard deviations are, respectively, $(-0.03 e,-0.18 e,-0.017 e),(0.1,0.5,0.9)$, and $(0.05,0.10,0.05)$. Ionic concentrations on both sides of the membrane are varied from 10 to $500 \mathrm{mM}$. Diffusion constants in the bulk are $D_{b, \mathrm{Na}^{+}}=1.33 \times 10^{-9} \mathrm{~m}^{2} / \mathrm{s}$ and $D_{b, \mathrm{Cl}^{-}}$ $=2.03 \times 10^{-9} \mathrm{~m}^{2} / \mathrm{s}$. Diffusion constants in the channel are $D_{\mathrm{Na}^{+}}=2.0 \times 10^{-10} \mathrm{~m}^{2} / \mathrm{s}$ and $D_{\mathrm{Cl}^{-}}=0.95 \times 10^{-10} \mathrm{~m}^{2} / \mathrm{s}$. The channel radius $=4 \AA$ and length $=30 \AA$ with a filling factor

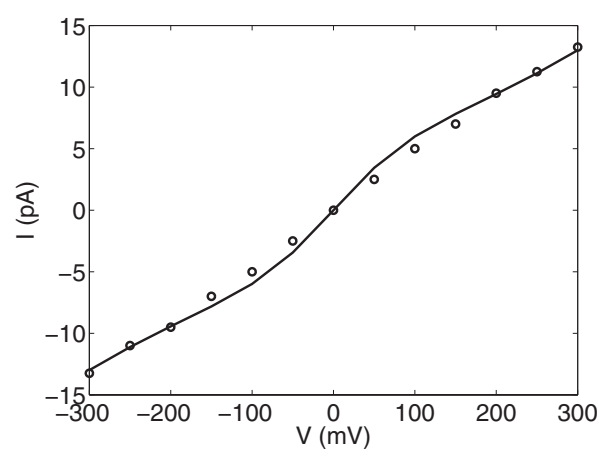

FIG. 5. Comparison of calculations with experiment. The full curve plots the current-voltage $(I-V)$ characteristics (solid line) calculated for a channel with $-0.227 e$ fixed charge in its middle, normally distributed with a standard deviation $\sigma=0.1$. The ionic concentrations on both sides of the membrane is $1 \mathrm{M}$, and the diffusion constants are $D_{\mathrm{Cs}^{+}}=D_{\mathrm{Cl}^{-}}=2.09 \times 10^{-10} \mathrm{~m}^{2} / \mathrm{s}$. The channel radius $=4 \AA$ and length $=30 \AA$, with a filling factor of 0.28 . The circle data points represent experiment [43].

of 0.1225 at $500 \mathrm{mM}$ and 0.25 at 10 and $100 \mathrm{mM}$. Hollerbach et al. $[45,46]$ used the spectral elements method to solve the 3D Poisson-Nernst-Planck equations for the Gramicidin channel. Portions of the bath were included into their simulations in order to have good boundary conditions. Their $I-V$ characteristics at several symmetric concentrations were also compared to the same $I-V$ curves measured experimentally by Andersen et al. [44] and were shown to be in good agreement. Next, we consider in more detail the dependence of the channel characteristics on other parameters.

\section{ANALYSIS OF THE CHANNEL PROPERTIES}

In this section, we investigate the influences of the membrane fixed charge and the bulk concentrations on channel permeation.

\section{A. Dependence on the channel fixed charge}

The effect of the fixed charge variation on the channel is determined by the calculation of the channel occupation

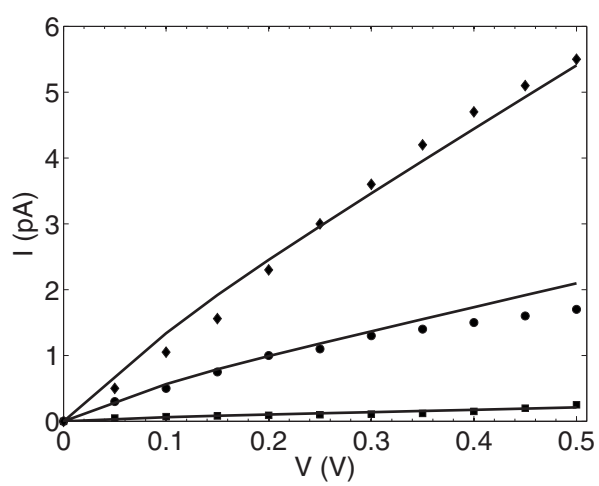

FIG. 6. Comparison of calculated current-voltage $(I-V)$ characteristics in the model (lines) with experimental data [44] for three different ionic concentrations, as follows: 10 (filled squares), 100 (filled circles), and $500 \mathrm{mM}$ (filled diamonds). 


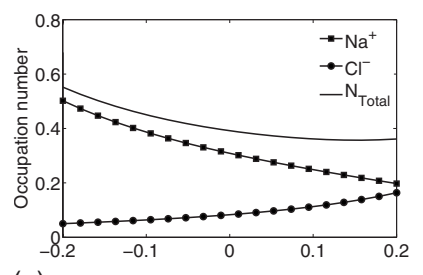

(a)

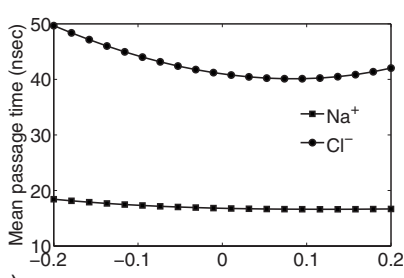

(b)

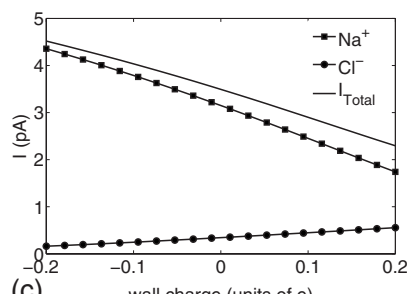

(c)

FIG. 7. Model calculations of (a) channel occupation number, (b) ionic mean passage time, and (c) ionic current, all as functions of the wall charge. The lines drawn through calculated points (filled circles for $\mathrm{Cl}^{-}$and filled squares for $\mathrm{Na}^{+}$) are guides to the eye, and the solid lines in (a) and (c) represent the total occupation number and the total current, respectively. The applied voltage is $300 \mathrm{mV}$. Ionic concentrations in the left and the right reservoirs are $c_{L}$ $=500 \mathrm{mM}$ and $c_{R}=40 \mathrm{mM}$, respectively. Diffusion constants are $D_{\mathrm{Na}^{+}}=D_{\mathrm{Cl}^{-}}=2.09 \times 10^{-10} \mathrm{~m}^{2} / \mathrm{s}$. The channel radius $=4 \AA$ and length $=30 \AA$ with a filling factor of 0.28 .

number, the mean passage time (MPT), and the current for each ion species as functions of the total fixed charge on the membrane wall (for given parameters of the fixed charge distribution). The occupation number $C_{i}$ of the channel for each ion species is found by integrating the corresponding concentration over the channel volume. For cylindrical geometry we have

$$
C_{i}=f_{s c} \pi a^{2} \int_{0}^{L} \tilde{n}_{i}(x) d x .
$$

Note the filling factor in the expression for the occupation number. In general the filling factor has to be introduced individually for each ion species; however, for simplicity, we use here one average filling factor.

In Fig. 7, the channel occupation number, the ions' MPT through the channel, and the channel current are estimated as functions of the charge on the wall as it is varied from $-0.2 e$ to $+0.2 e$. The fixed charge is distributed on the membrane wall according to Eq. (10). In these plots, the MPT through the channel is calculated as $\tau_{M P S}^{(i)}=C_{i} e / I_{i}$, where $I_{i}$ is the total current through the channel of the ion species $i$. Note that this number corresponds to the total time one ion spends on average in the channel during the transition (in our example from left to right). The $\tau_{M P S}^{(i)}$ differs from the usual mean first passage time by the factor $C_{i}$.

The calculations in Figs. 7-9 are carried out for concentrations in the left and the right baths of 500 and $40 \mathrm{mM}$, respectively, with an externally applied voltage of $300 \mathrm{mV}$. It can be seen from Fig. 7 that while the channel occupation number decreases for the cation as a function of the total wall charge, it increases for the anion. The conduction cur-
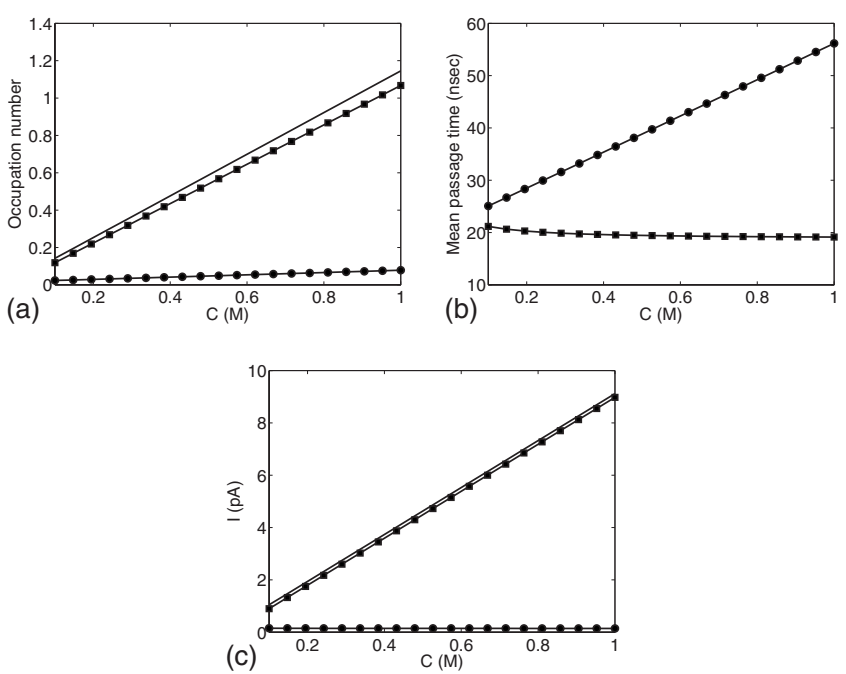

FIG. 8. Model calculations of (a) channel occupation number, (b) ionic mean passage time, and (c) ionic current, all as functions of bulk concentration. The lines drawn through calculated points (filled circles for $\mathrm{Cl}^{-}$and filled squares for $\mathrm{Na}^{+}$) are guides to the eye, and the solid lines in (a) and (c) represent the total occupation number and the total current, respectively. The external potential difference was set to $300 \mathrm{mV}$ with -0.227 e fixed charge in the middle of the channel normally distributed with standard deviation $\sigma=0.1$. The ionic concentration in the right reservoir is $c_{R}$ $=40 \mathrm{mM}$; that in the left reservoir is allowed to change. Diffusion constants are $D_{\mathrm{Na}^{+}}=D_{\mathrm{Cl}^{-}}=2.09 \times 10^{-10} \mathrm{~m}^{2} / \mathrm{s}$. The channel radius $=4 \AA$ and length $=30 \AA$ with a filling factor of 0.28 .

rent decreases as a function of fixed charge for $\mathrm{Na}^{+}$and increase for $\mathrm{Cl}^{-}$as the fixed charge increases and becomes positive. Note also that the total channel occupation numbers vary nonlinearly as functions of the fixed charge and are lower than 1 in the interval considered. The mean passage time shows a minimum for the anion and decreases for the cation as the wall charge is increased. The motion of $\mathrm{Cl}^{-}$ through the channel is slower than that of $\mathrm{Na}^{+}$. These dependences can be attributed to the charge affinity between the moving ions and the fixed charge distributed on the membrane wall. Estimation of the channel properties as a function of the channel fixed charge can be useful in gaining insight into the channel selectivity to specific ions as a result of the interactions between the ligand charge at the channel's binding sites and the permeant ions. Furthermore, selectivity be-
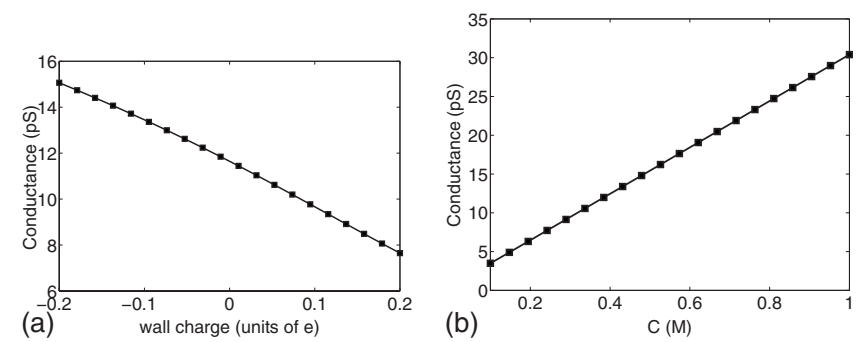

FIG. 9. Model calculations of (a) conductance as a function of the wall charge, using the parameters from Fig. 7, and (b) conductance as a function of concentration, using the parameters from Fig. 8. 
TABLE I. Equivalent conductivity at infinite dilution $\lambda^{0}$ and conductivity coefficients $G$ at $25^{\circ} \mathrm{C}$.

\begin{tabular}{lcc}
\hline \hline Species & $\begin{array}{c}z \lambda^{0} \\
\left(\mathrm{~cm}^{2} \mathrm{~S} / \mathrm{mol}\right)\end{array}$ & $\begin{array}{c}G \\
(\mathrm{~mol} / \mathrm{l})^{-1 / 2}\end{array}$ \\
\hline $\mathrm{Na}^{+}$ & 50.1 & 0.733 \\
$\mathrm{Cl}^{-}$ & 76.4 & 0.548 \\
\hline \hline
\end{tabular}

tween alike ions within the PNP formalism can be obtained by introducing a separate filling factor for each ion species in order to take account the ionic radius.

\section{B. Dependence on the bulk concentrations}

Figure 8 plots the channel occupation number, the ionic mean passage time, and the ionic current in the channel as functions of bulk concentration in the left-hand bath for an applied voltage of $300 \mathrm{mV}$. The concentration in the righthand bath was kept constant at $40 \mathrm{mM}$. The channel occupation number increases linearly with the bulk concentration. The same variations are observed for the cation currents in Fig. 8(c). As the bulk concentration increases, the $\mathrm{Cl}^{-}$current remain constant. This may be attributed to charge repulsion between the negatively charged protein $(-0.227 e)$ and the anion. We have ensured that the concentration changes investigated remain within the physiological range.

Figure 9 calculates the channel conductance as a function of the wall charge and the bulk concentration. It is found that channel conductance decays as the wall charge increase, whereas it increases as the bulk concentration increases.

\section{Diffusion-limited ion flow in the vicinities of the pore entrance}

The AR as described by Hall [18], $R_{\text {Hall }}=\frac{\rho}{4 a}$, is used as the reference for our calculation. Here, $a$ is the channel radius and $\rho$ is the resistivity of the solution. This corresponds to the resistance of the hemisphere close to the channel mouth, which is of the same order as the one of the remaining infinite sphere in the case of an uncharged membrane. The conductivity of the electrolyte is expressed as the weighted sum of the equivalent conductivities $\lambda_{i}$ and given by $\rho^{-1}$ $=\sum z_{i} \widetilde{c}_{i} \lambda_{i}$ [47]. The equivalent conductivities at $25^{\circ} \mathrm{C}$ are approximated by $\lambda_{i}=\lambda_{i}^{0} /\left(1+G_{i} \sqrt{I_{M}}\right)$, which characterizes the concentration well and remains reasonably accurate at concentrations near $1 \mathrm{~mol} / \mathrm{l}$. The quantity $I_{M}=\frac{1}{2} \sum_{i} z_{i}^{2} \widetilde{c}_{i}$ is the ionic strength (molar basis). The quantity $\lambda_{i}^{0}$ which is the equivalent conductivity of an ionic species at infinite dilution and the empirical coefficients $G_{i}$ are described by [48] and given in Table I.

We have estimated the AR to the channel by considering the existence of voltage drops along the left and the right mouths of the channel given, respectively, by $\widetilde{\phi}_{L}=\widetilde{\phi}\left(\widetilde{x}_{0}\right)$ $-\widetilde{\phi}_{L}^{\infty}$ and $\widetilde{\phi}_{R}=\widetilde{\phi}_{R}^{\infty}-\widetilde{\phi}\left(\widetilde{x}_{1}\right)$. The access resistances in the leftand the right-hand mouths are given, respectively, by $R_{L}$ $=\widetilde{\phi}_{L} / \widetilde{I}^{c h}$ and $R_{R}=\widetilde{\phi}_{R} / \widetilde{I}^{c h}$, where $\widetilde{I}^{c h}$ is the total current through the channel. The total AR to the channel is calcu-

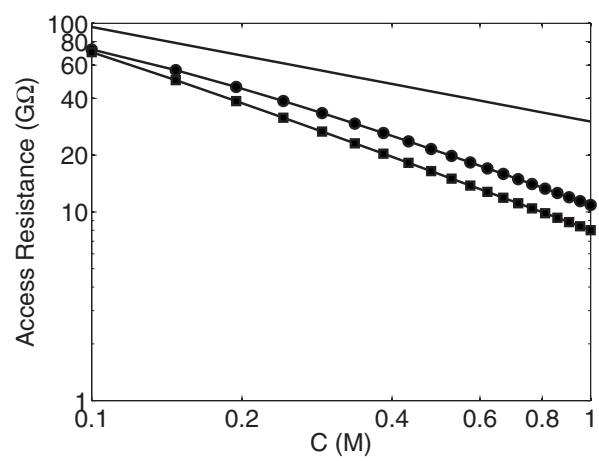

FIG. 10. AR calculated as a function of bulk concentration for a channel of radius $4 \AA$ and length of $30 \AA$, embedded in a negatively charged membrane, with charge $-0.227 e$ distributed with a one-mode Gaussian distribution of standard deviation $\sigma=0.1$, with the charges concentrated at the channel center, for a $300 \mathrm{mV}$ external potential difference. The lines drawn through calculated points (filled circles for uncharged channel and filled squares for charged channel) are guides to the eye, and the solid line represents the Hall AR. The ionic concentration in the right reservoir is $c_{R}=40 \mathrm{mM}$; that in the left reservoir is allowed to change. Diffusion constants and filling factor are the same as in Fig. 8.

lated as $R_{T}=\left|R_{L}+R_{R}\right|$. We study diffusion-limited ion flow in the vicinities of the pore entrance. The AR to the channel as a function of the bulk concentration in the left bath is calculated for uncharged and charged channels as shown in Fig. 10. Our estimated ARs for uncharged and charged channels remain below the AR estimated using the Hall formula. The fixed charge is distributed on the channel surface and mainly concentrated at the channel center, corresponding to a charged membrane with binding site at its center. In the presence of the fixed charge on the membrane surface, the AR to the channel decreases as the concentration increases, leading to flows much larger than the diffusion of ions for an uncharged channel. At higher concentration, the AR of the charged channel is smaller than that of an uncharged channel. High current flow in the presence of fixed charge on the membrane wall may result in the current saturation observed at high concentration.

\section{SUMMARY AND CONCLUSIONS}

We have introduced a self-consistent analytic approach for the calculation of the ion current through a channel and the access resistance at the channel mouth as a function of the applied potential and the bulk concentrations. In this approach the PNP solution in the channel is coupled to the PBNP solution in the bulk by matching the corresponding boundary conditions at the channel mouth in a self-consistent way and by introducing the "filling factor" as an additional fitting parameter that takes into account the radial distribution of current density in the channel. We note that this factor is introduced as a simple approximation of the free-energy contribution due to the potential of the mean force in the channel. We note also that factors of this type are common in semiconductor theories (cf. the ideality factor in the diode equation [42]) that are used to model conduction in ion chan- 
nels [33]. The method allows for the calculation of the currents for an arbitrary number of monovalent ion species that have different diffusion constants in the channel and in the bulk. It is applied to fit experimental $I-V$ characteristics of the Gramicidin A channel for various concentrations, yielding good quantitative agreement with the experimental results.

It is further shown that the changes in experimental $I-V$ characteristics as a function of bulk concentrations can be successfully reproduced by the method. The corresponding changes in the filling factor can be attributed to the effect of saturation [49].

Given that the 1D PNP solution was earlier applied successfully to the analysis of experimental $I-V$ characteristics in many ion channels [11-15], the scope of the present study also goes beyond the Gramicidin channel. In particular, with a subtle transformation of the channel structure, the work could be extended to other types of ion channel such as the $L$-type $\mathrm{Ca}^{2+}$ and $\mathrm{Na}^{+}$channels. This implies adjustment of the concentrations and the fixed charge on the channel wall. Because the filling factor allows for the ionic radius to be taken into account, it can be introduced for each ion species separately and the results obtained can also be used to gain further insight into the ion channel selectivity mechanisms that distinguish between alike ions.

During the preparation of this paper, another interesting analytical approximation of the solution of the PNP equations appeared in press [33]. In this approximation, which follows closely known asymptotic techniques of semiconductor physics [50], matching of the solution and the boundary layers at the channel entrances is not required. However, the resultant analytical solution is discontinuous at the channel boundaries and for this reason is not well suited to the estimation of the access resistance discussed in the present paper. Besides, the approximation relies on charge neutrality, which was shown earlier to be a less accurate approximation to the numerical solution of the PNP in the bulk [21] than the linear approximation adopted here. The results [33] obtained for the constant charge density on the channel walls are not compared with experimental data. It will, however, be of interest to compare the two analytical approximations in the future.

The approach we have introduced can be further improved in a number of ways. First, the boundary conditions can be improved using, e.g., the theoretical discussion in $[22,51]$. More importantly, the approach opens the possibility of an analytical treatment of the subtle details of the potential of the mean force revealed through molecular-dynamics simulations (see, e.g., [30]) but at the level of continuous PNP approximation. In particular, extending the idea of the filling factor, and including explicitly the PMF due to the wall vibrations and ion-wall interaction, will lead to a timedependent Nernst-Planck equation with $\widetilde{\psi}_{j}^{P M F}$ specific for each ion species, thereby opening up the possibility of analytic insight into the selectivity of ion channels between alike ions. A model using density-functional theory [52] to take care of finite-size effects could also be used to patch the bulk solution.

\section{ACKNOWLEDGMENTS}

The work was supported by the Engineering and Physical Sciences Research Council (U.K.), the Russian Foundation for Fundamental Science, INTAS, and ESF.
[1] B. Hille, Ionic Channel of Excitable Membranes, 3rd ed. (Sinauer Associates, Sunderland, MA, 2001).

[2] R. S. Eisenberg, Contemp. Phys. 39, 447 (1998).

[3] D. Stein, M. Kruithof, and C. Dekker, Phys. Rev. Lett. 93, 035901 (2004).

[4] K. E. Cooper, P. Y. Gates, and R. S. Eisenberg, J. Membr. Biol. 106, 95 (1988).

[5] D. P. Chen, J. Lear, and R. S. Eisenberg, Biophys. J. 72, 97 (1997).

[6] B. Corry, T. W. Allen, S. Kuyucak, and S.-H. Chung, Biophys. J. 80, 195 (2001).

[7] B. Roux, Biophys. J. 71, 3177 (1996).

[8] V. Barcilon, SIAM J. Appl. Math. 52, 1391 (1992).

[9] V. Barcilon, D. P. Chen, and R. S. Eisenberg, SIAM J. Appl. Math. 52, 1405 (1992)

[10] S. Kuyucak, M. Hoyles, and S.-H. Chung, Biophys. J. 74, 22 (1998).

[11] D. P. Chen, V. Barcilon, and R. S. Eisenberg, Biophys. J. 61, 1372 (1992).

[12] R. Elber, D. P. Chen, D. Rojewska, and R. Eisenberg, Biophys. J. 68, 906 (1995).

[13] W. Nonner and B. Eisenberg, Biophys. J. 75, 1287 (1998).

[14] D. P. Chen et al., Biophys. J. 76, 1346 (1999).

[15] R. S. Eisenberg, J. Membr. Biol. 171, 1 (1999).
[16] S. M. Bezrukov and I. Vodyanoy, Biophys. J. 64, 16 (1993).

[17] J. Song, C. A. S. A. Minetti, M. S. Blake, and M. Colombini, Biophys. J. 76, 804 (1999)

[18] J. E. Hall, J. Gen. Physiol. 66, 531 (1975).

[19] B. Hille, Ionic Channel of Excitable Membranes (Sinauer Associates, Sunderland, MA, 1992).

[20] M. S. P. Sansom and I. D. Kerr, Biophys. J. 69, 1334 (1995).

[21] A. Peskoff and D. M. Bers, Biophys. J. 53, 863 (1988).

[22] V. Levadny, V. Aguilella, and M. Belaya, Biochim. Biophys. Acta 1368, 338 (1998).

[23] M. Aguilella-Arzo, V. M. Aguilella, and R. S. Eisenberg, Eur. Biophys. J. 34, 314 (2005).

[24] M. G. Kurnikova, R. D. Coalson, P. Graf, and A. Nitzan, Biophys. J. 76, 642 (1999).

[25] G. Moy, B. Corry, S. Kuyucak, and S.-H. Chung, Biophys. J. 78, 2349 (2000).

[26] A. Singer, Z. Schuss, D. Holcman, and R. S. Eisenberg, J. Stat. Phys. 122, 437 (2006).

[27] D. Gillespie and R. S. Eisenberg, Phys. Rev. E 63, 061902 (2001).

[28] D. Gillespie and R. S. Eisenberg, Eur. Biophys. J. 31, 454 (2002).

[29] R. D. Coalson and M. G. Kurnikova, in Biological Membrane Ion Channels, Biological and Medical Physics, Biomedical 
Engineering, edited by S.-H. Chung, O. S. Andersen, and V. Krishnamurthy (Springer, New York, 2007), Chap. 13, pp. 449-484.

[30] S. Y. Noskov, S. Berneche, and B. Roux, Nature (London) 431, 830 (2004).

[31] D. Luchinsky et al., J. Phys.: Conf. Ser. 142, 012049 (2008).

[32] D. P. Chen and R. S. Eisenberg, Biophys. J. 65, 727 (1993).

[33] A. Singer, D. Gillespie, J. Norbury, and R. Eisenberg, Eur. J. Appl. Math. 19, 541 (2008).

[34] D. P. Chen and R. S. Eisenberg, Biophys. J. 64, 1405 (1993).

[35] S. McLaughlin, Annu. Rev. Biophys. Biophys. Chem. 18, 113 (1989).

[36] D. P. Chen, J. Tang, and R. S. Eisenberg, Nanotechnology 2, 64 (2002).

[37] R. S. Eisenberg, M. M. Klosek, and Z. Schuss, J. Chem. Phys. 102, 1767 (1995).

[38] C. Gardiner, Handbook of Stochastic Methods for Physics and Chemistry, Springer Series in Synergetics No. 13 (Springer, New York, 1996).

[39] H. K. Gummel, IEEE Trans. Electron Devices 11, 455 (1964).

[40] R. S. Eisenberg, J. Membr. Biol. 150, 1 (1996).

[41] H. Monoi, Biophys. J. 59, 786 (1991).
[42] S. Selberherr, Analysis and Simulation of Semiconductor Devices (Springer-Verlag, Wien, NY, 1984).

[43] S. Oiki, R. E. Koeppe II, and O. S. Andersen, Biophys. J. 66, 1823 (1994).

[44] O. S. Andersen, R. E. Koeppe, and B. Roux, IEEE Trans. Nanobiosci. 4, 10 (2005)

[45] U. Hollerbach, D. P. Chen, D. Busath, and R. S. Eisenberg, Langmuir 16, 5509 (2000).

[46] U. Hollerbach, D. P. Chen, and R. S. Eisenberg, J. Sci. Comput. 16, 373 (2001).

[47] J. O. Bockris and A. K. N. Reddy, Modern Electrochemistry, 2nd ed. (Plenum Press, New York, 1998).

[48] K. A. Snyder, X. Feng, B. D. Keen, and T. O. Mason, Cem. Concr. Res. 33, 793 (2003).

[49] B. Corry, S. Kuyucak, and S.-H. Chung, Biophys. J. 78, 2364 (2000).

[50] P. A. Markowich, C. A. Ringhofer, and C. Schmeiser, Semiconductor Equations (Springer-Verlag, Wien, NY, 1990).

[51] B. Eisenberg and W. Liu, SIAM J. Math. Anal. 38, 1932 (2007).

[52] D. Gillespie, W. Nonner, and R. S. Eisenberg, J. Phys.: Condens. Matter 14, 12129 (2002). 